\title{
Plunging ranula: An interesting case report
} \author{
Zaheer Kota $^{1}$ \\ ${ }^{1}$ Oral \& Maxillofacial Surgery, College of Dentistry, King Khalid University, Abha, KSA \\ ${ }^{2}$ Oral Pathology, College of Dentistry, King Khalid University, Abha, KSA \\ ${ }^{3}$ Oral Medicine \& Radiology, College of Dentistry, King Khalid University, Abha, KSA \\ Email: *kamranbokhari@gmail.com
}

Abdul Bagi Mustafa ${ }^{1}$, Kamran Bokhari ${ }^{1 *}$, Master Luqman², Mohammad Shahul Hameed ${ }^{3}$,

Received 28 January 2013; revised 3 March 2013; accepted 15 March 2013

\begin{abstract}
Background: Ranula is a retention cyst of the sublingual gland, which enlarges progressively and extends into the surrounding soft tissues. The name "ranula" is derived from the Latin word "rana" meaning "frog". These represent for $6 \%$ of all oral sialocysts. Two variants have described in the literature: simple oral ranula and the deep diving or plunging ranula. The plunging ranula has the potential to spread into deeper parapharyngeal spaces and presents a diagnostic dilemma due to its clinical similarity with other neck masses such as cystic hygroma, thyroglossal duct cyst, intramuscular hemangioma, cystic/neoplastic thyroid disease, branchial cyst. We report a case of large oral ranula involving the floor of mouth treated successfully by surgical excision. Case Report: This case report involves an unusually large ranula involving right floor of the mouth in a 30-year-old female patient. Following detailed clinical examination, radiological interpretation and histopathological diagnosis, surgical excision was performed along with the involved sublingual gland. The patient was followed up on a regular basis and was disease free. Summary and Conclusion: The best treatment for an oral/simple ranula is excision of the lesion along with the involved gland. This case report involving the floor of the mouth, is interesting and unique due to its unusually large size and the complete treatment rendered by us i.e., excision of the lesion along with the involved sublingual gland and the subsequent confirmation with the histopathological findings.
\end{abstract}

Keywords: Ranula; Salivary Gland; Oral Swelling

\section{INTRODUCTION}

Ranula is a retention cyst of the sublingual gland, which

*Corresponding author. enlarges progressively and extends into the surrounding soft tissues. Two variants have been described: a superficial or oral ranula and a cervical or plunging ranula [1]. Simple ranulas remain confined to the sublingual space, whereas diving ones extend beyond it [2]. Ranulas superior to the mylohyoid muscle appear as a translucent bluish swelling under the tongue, resembling a frog's underside [3]. Though the exact prevalence of plunging ranulas is not known, simple ranulas have been reported to have a prevalence rate of 0.2 cases for every thousand people by various authors [3-5]. Suresh BV and Vora SK reported a male to female ratio as 1:1.3 without significant side preference [6]. Primary etiology of these lesions is due to partial obstruction of a sublingual duct which leads to the formation of an epithelial-lined retention cyst [5]. Congenital anomalies such as duct agenesis, hypoplasia of the sublingual gland and trauma causing direct damage to the duct or deeper areas of the sublingual gland are few of the other etiological factors described in the literature $[5,7]$.

Whereas oral/simple ranulas do not pose much diagnostic challenge as they present themselves as a swelling under the floor of the mouth, correct differentiation of a diving/plunging ranula is critical. Plunging ranulas typically extend posteriorly beyond the free edge of the mylohyoid muscle thus sometimes encroaching the submandibular and parapharyngeal spaces [2]. This poses diagnostic challenge to differentiate from other neck swellings especially from cystic hygroma. We present an unusually large case of oral ranula involving floor of the mouth in a 30 -year-old female patient which was successfully excised along with the involved sublingual gland.

\section{CASE REPORT}

A 30-year-old female patient reported to our center with a chief complaint of chronic swelling in the right floor of the mouth since 3 months (Figure 1). On clinical examination, a bluish well circumscribed swelling measuring 


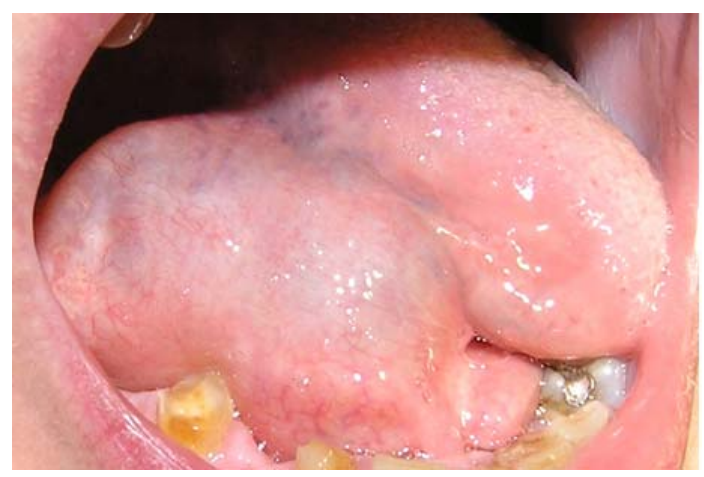

Figure 1. Clinical appearance of the swelling.

approximately 5*5 centimeters was noticed. Medially, the swelling extended till the midline of the floor of the mouth and the lateral extent was till the lingual vestibule. Posteriorly, the swelling limited unilaterally to an imaginary line drawn from the right first molar to the other side first molar thus confirming that the swelling did not extend beyond the posterior margin of the mylohyoid muscle into deeper spaces. Tongue was displaced towards the left side due to the swelling. Patient had difficulty in mastication and swallowing due to the large size of the swelling obstructing tongue movements. Careful examination of the right submandibular and sublingual ducts revealed patency. There was limited flow of the saliva from the right ductal openings of the respective glands as compared to the left ones. The right and left submandibular and sublingual glands were stimulated by making the patient chew a sugar candy and with subsequent milking of the glands. Bimanual palpation of the right sublingual gland revealed slight tenderness as compared to the left one. There was no history of chronic cheek bite or irritation. No significant extraoral swelling or asymmetry was evident. The swelling was soft in consistency and non-tender on palpation. Mucosa over the swelling was normal with absence of any ulcerations or sinus discharge. There were no other secondary changes involved like paresthesia or cervical lymphadenopathy.

Radiographic examination was not significant with absence of any calcifications. Aspiration was performed under topical anesthesia and yielded collection of thick mucus like aspirate which was subjected to cytological examination. Initial diagnosis of ranula was made on the basis of clinical appearance and cytological examination. The patient was then advised to undergo Color Doppler of the lesion to rule out any active feeder vessel characteristic of hemangioma. Ultrasound findings were negative in terms of vascularity or a feeder vessel. Based on the clinical, histological and ultra-sound findings, treatment was planned for excision of the lesion along with the involved sublingual gland. The lesion was approached intraorally through a mucosal incision placed over the lesion (Figure 2). Careful dissection in the sub-mu-

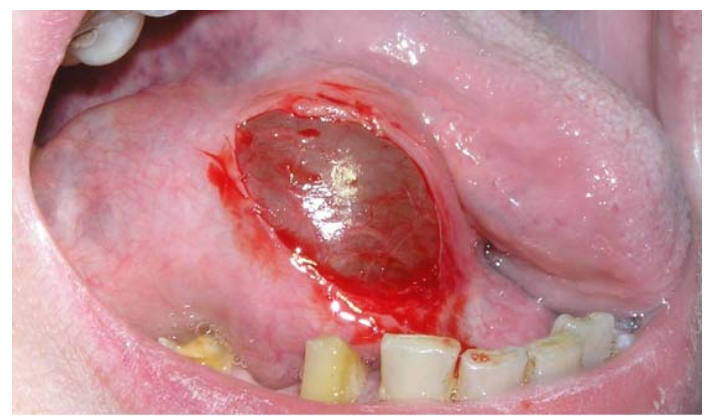

Figure 2. Incision.

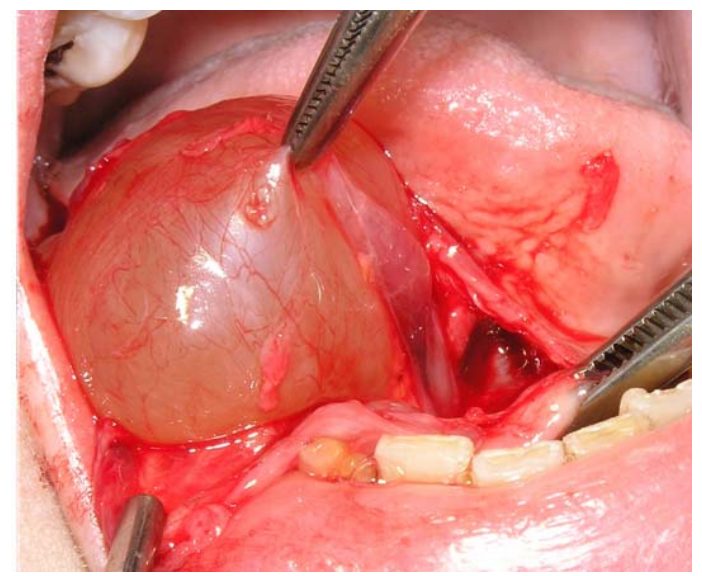

Figure 3. Exposed ranula.

cosal plane revealed a well encapsulated soft swelling which was friable but could be separated from the surrounding connective tissue and muscle plane (Figure 3). The swelling on the deeper aspect was extending towards the sublingual gland. The submandibular and sublingual ducts were separated from the dissection plane. Sublingual gland was then dissected out along with the duct and then completely excised (Figure 4). Complete hemostasis was achieved and primary closure performed. The excised specimen (Figure 5) was sent for histological examination which confirmed the diagnosis of ranula. (Figure 6) shows mucous laden macrophages along with damaged and dilated minor salivary gland ducts in the connective tissue. Few normal mucousacini and chronic inflammatory cells are also seen.

There were no specific complaints post-operatively and the patient was discharged three days post-operatively. The patient was disease free without any recurrence during the follow-up period.

\section{DISCUSSION}

"A ranula is a mucus filled cavity, a mucocele, in the floor of the mouth in relation to the sublingual gland" [5]. The name "ranula" is derived from the Latin word "rana" meaning "frog". These represent for $6 \%$ of all oral sialocysts. Although there is no specific sex predilection for 


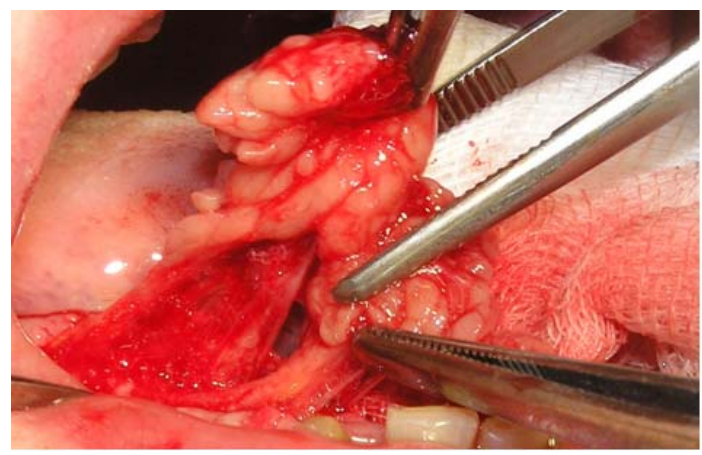

Figure 4. Dissection of sublingual gland.

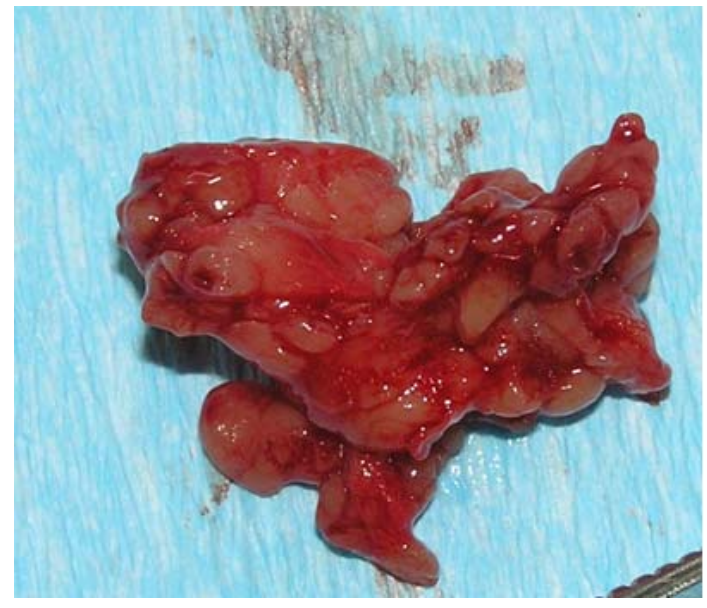

Figure 5. Excised sublingual gland.

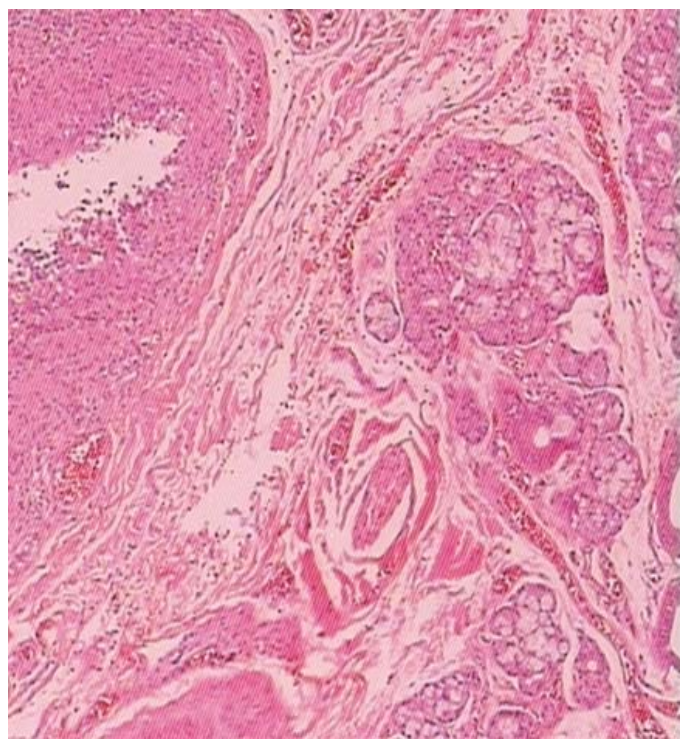

Figure 6. Histopathological findings.

ranulas, Neil EI et al., in their case report and review of literature involving a plugingranula stated that ranulas are more common in females [8].

Arunachalam P. et al., in 2010 reported two cases of recurrent plunging ranula and stated that ranula is an ex- travasation cyst in the floor of the mouth which develops from extravasation of mucus after trauma to the sublingual gland or obstruction of the duct [7]. The pathophysiology involved in extravasation is hypertension in the duct due to obstruction leading to acinar rupture in the salivary gland and then extravasation of the mucus. More recently in 2012, Suresh B.V. and Vora S.K. have extensively described 3 mechanisms for the origin of ranula in the neck: 1) Sublingual gland projecting through the mylohyoid or an ectopic sublingual gland may exist on the cervical side of mylohyoid; 2) Dehiscence in the anterior part of the mylohyoid muscle providing a path for ranula into the floor of the mouth; 3) A duct from the sublingual gland may join the submandibular gland or its duct, allowing ranulas to form in continuity with the submandibular gland [6]. Plunging ranulas are mucus retention cysts from sublingual gland or duct with extension into deeper spaces. Macdonald A.J. et al., in 2003 reported a giant ranula of the neck and stated that giant ranula is one that significantly involves the parapharyngeal space in addition to the submandibular space which makes differentiation from other cystic neck masses, particularly cystic hygroma difficult [2]. Thyroglossal duct cyst, intramuscular hemangioma, cystic/neoplastic thyroid disease, branchial cyst, submandibular sialadenitis, laryngocele, dermoid cyst and infectious cervical lymphadenopathy like tuberculosis, Epstein-Barr virus are other conditions which must be ruled out in before confirming plunging/diving ranula. Guruprasad Y. and Chauhan D.S. reported a case of cervical cystic hygroma in a six-year-old male patient and discussed cystic hygromas as congenital malformations of the lymphatic system with most of them being diagnosed within the first 2 years of life [9]. It is also to be noted that majority of the cystic hygromas develop within the posterior triangle of the neck. Jain H. et al., in 2012 reported a case of giant sublingual dermoidcyst in the floor of the mouth [10]. They elaborated dermoid cysts as congenital lesions covered with epithelium showing keratinization and presenting identifiable dermal appendices. A detailed history, clinical examination and histological diagnosis is imperative to differentiate ranulas from few of the above discussed lesions.

Effective treatment involves excision along with the involved sublingual gland [11]. $\mathrm{CO}_{2}$ laser as a treatment modality has been used in few cases to vaporize ranulas [5]. In rare cases, radiation therapy has been instituted as a treatment modality. Rho M.H. et al., evaluated the efficacy of OK-432 (lyophilized mixture of a low virulence strain of Streptococcus pyogenes incubated with benzylpenicillin) sclerosis as an alternative to surgery in the treatment of plunging ranula's in 31 patients [12]. They concluded that OK-432 sclerotherapy of plunging ranula is a safe and potentially curative procedure that may be 
used as a primary treatment for plunging ranula before considering surgery. In our view, the best treatment for an oral/simple ranula is excision of the lesion along with the involved gland and other treatment modalities should be considered for non-accessible/plunging ranula's. This case report by us involving the floor of the mouth, is interesting and unique due to its unusually large size and the complete treatment rendered by us i.e., excision of the lesion along with the involved sublingual gland and the subsequent confirmation with the histopathological findings.

\section{REFERENCES}

[1] De Visscher, J.G., Vander Wal, K.G. and de Vogel, P.L. (1989) The plunging ranula. Pathogenesis, diagnosis and management. Journal of Cranio-Maxillofacial Surgery, 17, 182-185.

[2] Macdonald, A.J., Salman, K.L. and Harnsberger, H.R. (2003) Giant ranula of the neck: Differentiation from cystic hygroma. American Journal of Neuroradiology, 24, 757-761.

[3] Kalra, V., Mirza, K. and Malhotra, A. (2011) Plunging ranula: Case report. Radiology Case, 5, 18-24.

[4] Bahnassy, M. (2009) A huge ranula. OMJ, 24, 306-307. doi:10.5001/omj.2009.63
[5] Gupta, A. and Karjodkar, F.R. (2011) Plunging ranula: A case report. ISRN Dentistry, 2011, 1-5. doi: $10.5402 / 2011 / 806928$

[6] Suresh, B.V. and Vora, K.S. (2012) Huge plunging ranula. Journal of Oral and Maxillofacial Surgery, 11, 487-490.

[7] Arunachalam, P. and Priyadharshini, N. (2010) Recurrent plunging ranula. Journal of Indian Association of Pediatric Surgeons, 15, 36-38.

[8] Langlois, N.E.I., BChir, M.B. and Kolhe, P. (1992) Plunging ranula: A case report and a literature review. Human Pathology, 23, 1306-1308. doi:10.1016/0046-8177(92)90300-R

[9] Guruprasad, Y. and Chauhan, D.S. (2012) Cervical cystic hygroma. Journal of Maxillofacial and Oral Surgery, 11, 333-336. doi:10.1007/s12663-010-0149-x

[10] Jain, H., Sing, S. and Singh, A. (2012) Giant sublingual dermoid cyst in floor of the mouth. Journal of Maxillofacial and Oral Surgery, 11, 235-237. doi:10.1007/s12663-010-0093-9

[11] Harrison, J.D. (2010) Modern management and pathophysiology of ranula: Literature review. Head and Neck, 32, 1310-1320. doi:10.1002/hed.21326

[12] Rho, M.H., Kim, D.W., Kwon, J.S., Lee, S.W., Sung, Y.S., Song, Y.K., Kim, M.G. and Kim, S.G. (2006) OK-432 sclerotherapy of plunging ranula in 21 patients: It can be a substitute for surgery. American Journal of Neuroradiology, 27, 1090-1095. 